\section{Elterngeld - Für die Pflege der Eltern}

\begin{abstract}
- Auch ein Jahr nach Einführung der neuen Regelungen zur besseren Vereinbarkeit von Familie, Pflege und Beruf glaubt die große Mehrheit der Erwerbstätigen in Deutschland nicht, dass sich Beruf und Pflege gut vereinbaren lassen. Laut einer repräsentativen Befragung des Zentrums für Qualität in der Pflege (ZQP) beurteilen $80 \%$ der erwerbstätigen Deutschen deren Vereinbarkeit als schlecht. Allerdings meinen 73\% der Befragten, dass der Rechtsanspruch auf ein „Pflegenden-Geld" pflegende Angehörige erheblich entlasten würde und so zur besseren Vereinbarkeit von Beruf und Pflege beitragen könnte. Ein teilweise diskutiertes „Pflegenden-Geld“ wäre eine dem Elterngeld vergleichbare Unterhaltsleistung.

In Analogie zum Elterngeld könnten sich Berufstätige für die Pflege eines Angehörigen bis zu zwölf Monate von ihrem Job freistellen lassen. Während dieser Auszeit erhielten sie etwa zwei Drittel ihres vorherigen Einkommens (mindestens 300 Euro, höchstens 1.800 Euro), bei gleichzeitigem Rückkehrrecht in die Vollerwerbstätigkeit.
\end{abstract}

Nach Ansicht der Befragten könnte dadurch auch die Bereitschaft, Pflege zu übernehmen, wesentlich erhöht werden. Zudem glaubt mehr als ein Drittel der Berufstätigen, dass mehr Männer bereit wären, Pflegeverantwortung zu übernehmen.

Bleibt die Frage, ob solch ein finanzieller Anreiz, nicht auch Risiken birgt? Denn kritische Pflegesituationen - wie die Vernachlässigung oder Missachtung eines pflegebedürftigen Menschen - drohen auch dort zu entstehen, wo überwiegend finanzielle und nicht fürsorgliche Motive zur Übernahme einer Pflegeaufgabe bewegen. Zudem sind die Kosten des "Pflegenden-Geldes” nur schwer kalkulierbar. In den wissenschaftlichen Untersuchungen gehen Experten teilweise von einer durchschnittlich achtjährigen Dauer der häuslichen Unterstützung aus. Ein „Pflegenden-Geld“ müsste also in vielen Fällen deutlich länger als das Elterngeld gezahlt werden - Unternehmen entsprechend lange auf ihre Mitarbeiter verzichten.

www.zqp.de
Quality Award

Pflegephilosophie nach Silviahemmet ${ }^{\circledR}$

- Der bpa (Bundesverband privater Anbieter sozialer Dienste e.V.) vergibt alle zwei Jahre den „bpa Quality Award“ für innovative Projekte, die für eine Verbesserung der Qualitätsorientierung sorgen.

Bei der Preisverleihung in Dresden wurde der Award im Rahmen der Bundesmitgliederversammlung an das St. Anna-Stift Kroge verliehen. Die deutschlandweit erste, vollstationäre Pflegeeinrichtung für demenziell Erkrankte nach Silviahemmet ${ }^{\circledR}$, fokussiert sich nicht nur auf die Pflege der Bewohner, sondern berücksichtigt auch die Bedürfnisse der Angehörigen und Pflegekräfte. Silviahemmet ${ }^{\oplus}$ (Hemmet schwed. Zuhause) ist eine von Königin Silvia initiierte Pflegephilosophie - die Mutter der schwedischen Königin erkrankte an Alzheimer -, die sich speziell an Demenzpatienten richtet. Sie basiert auf den vier Säulen Symptomkontrolle, Teamarbeit, Angehörigenunterstützung und Kommunikation/Beziehungsarbeit.

www.zerhusenbloemer.de

\section{Online-Patientenverfügung Innovativ \& einfach}

— Die Deutsche Gesellschaft für Vorsorge stellt ab sofort ein Online-Portal bereit, über das individuelle Patientenverfügungen einfach und kostengünstig erstellt werden können. Das Portal bietet nicht nur Hilfe bei der Erstellung medizinisch, juristisch und ethisch fundierter Vorsorgedokumente, sondern auch eine sichere Verwahrung mit einem Notfallzugriff rund um die Uhr. Der Nutzer wird einfach und verständlich durch alle relevanten Fragestellungen geführt. „meinepatientenverfügung.de" unterstützt auf der Grundlage eines interaktiven Online-Interviews bei der Information und Meinungsbildung sowie der Erstellung fundierter und möglichst zweifelsfreier Verfügungen.

www.meinepatientenverfügung.de

\title{
Berufsbekleidung \\ Aus für den langärmeligen Kasack
}

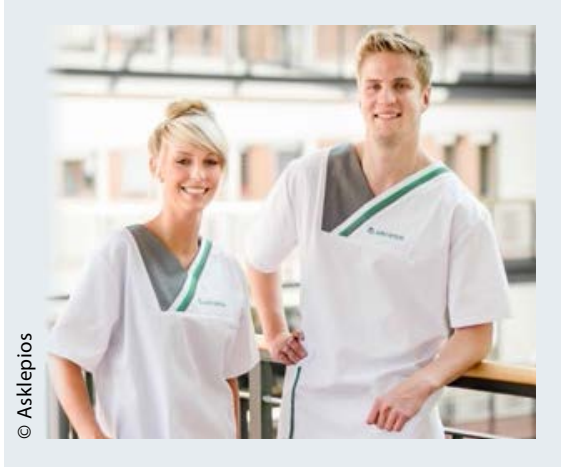

— Der Klinikkonzern Asklepios kleidet Ärzte und Pflegepersonal in seinen rund 100 Einrichtungen neu ein. Der klassische langärmlige Kasack wird von April an ausgemustert, kurze Ärmel sind angesagt - auch für Ärzte. Damit reagiere man auf die Sorge der Patienten vor einer Ansteckung mit multiresistenten Keimen, erklärte Konzerngeschäftsführer Kai Hankeln. Asklepios ist nach eigenen Angaben der erste große Klinikbetreiber in Deutschland, der sich zu diesem Schritt entschlossen hat. Der Konzern folge damit Empfehlungen des Robert Koch-Instituts und der Weltgesundheitsorganisation (WHO). Da gerade die Ärmel stark mit Keimen belastet sind, ist kurzärmlige Kleidung in Operationssälen und auf Intensivstationen längst Standard. Examinierte Pflegekräfte und Schwestern tragen nun einen Kasack mit grünen Streifen am Revers und grauem V-Ausschnitt.

www.asklepios.de 